\title{
Late onset bile duct stricture caused by iatrogenic injury during laparoscopic cholecystectomy, mimicking cholangiocellular carcinoma
}

Extrahepatic cholangiocellular carcinomas are relatively rare malignant tumors. Although recent years have seen advances in diagnosis and treatment, cholangiocellular carcinomas are usually clinically silent or associated with nonspecific symptoms in the early stages; therefore, most tumors are diagnosed late, when they are inoperable [1]. The diagnosis can be especially challenging in patients with chronic cholangitis or previous hepatobiliary and pancreatic surgery.

We report an unusual case of a 49-yearold woman who presented with painless jaundice. In the past half year she had experienced loss of appetite and weight. Preoperative imaging including magnetic resonance imaging, magnetic resonance cholangiopancreatography (MRCP), endoscopic retrograde cholangiography (ERC), and computed tomography suggested cholangiocellular carcinoma (Bismuth type II/IIIb) ( Figs. 1, 2). Brush cytology at ERC was not meaningful but a granulomatous tumor at the hilum with retained nonabsorbable sutures was found intraoperatively ( Fig. 3). The final histological examination yielded a benign disease described as periductular fibrosis and chronic inflammation. Following a biliodigestive anastomosis, the patient's status improved uneventfully ( $\bullet$ Fig. 4 ). A retrospective review revealed an iatrogenic bile duct injury sustained during laparoscopic cholecystectomy 9 years previously, with end-to-end repair carried out in the same session. The patient was unaware of the earlier iatrogenic lesion.

Preoperative findings can suggest malignancy even in benign disease. Correct diagnosis will depend on a careful preoperative diagnostic workup including a detailed history, with special attention to previous surgeries. Definitive diagnosis will usually require surgical exploration, as chronic biliary inflammation or obstructive cholestasis is a known risk factor for cholangiocellular carcinomas. Nonetheless, up to $15 \%$ of suspicious tumors are benign lesions [2,3]. Long, benign bile duct strictures are repaired with a biliodigestive anastomosis ( $\bullet$ Fig. 4) but they do not require extended lymphadenectomy, which is associated with increased

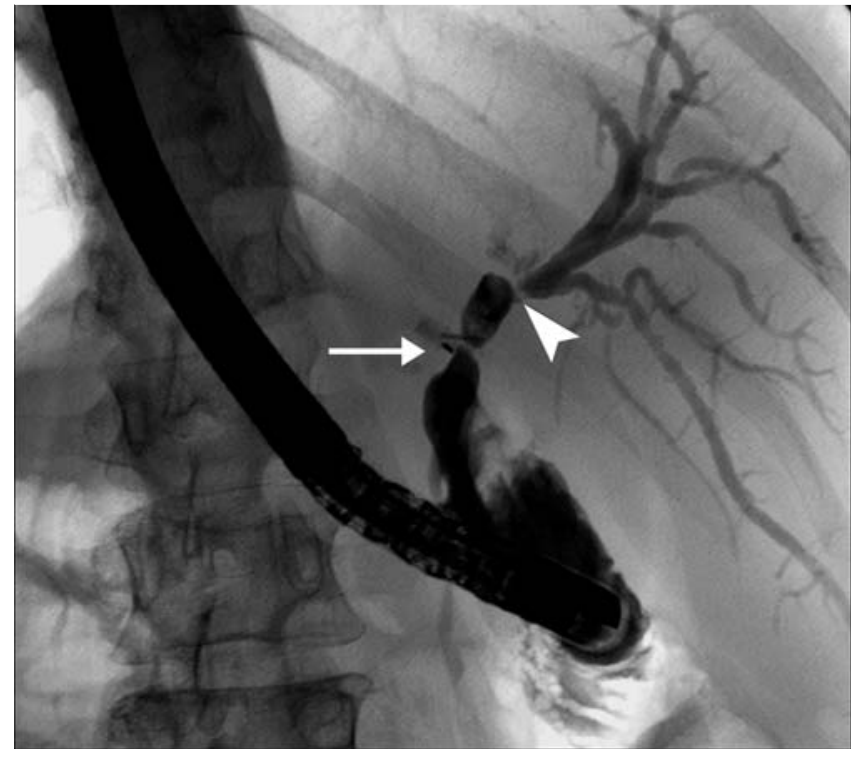

Fig. 1 Preoperative endoscopic retrograde cholangiography (ERC; posteroanterior view) showing a stricture at the cystic stump (arrow) and a stricture at the hilum (arrowhead) obstructing the left hepatic bile duct system.

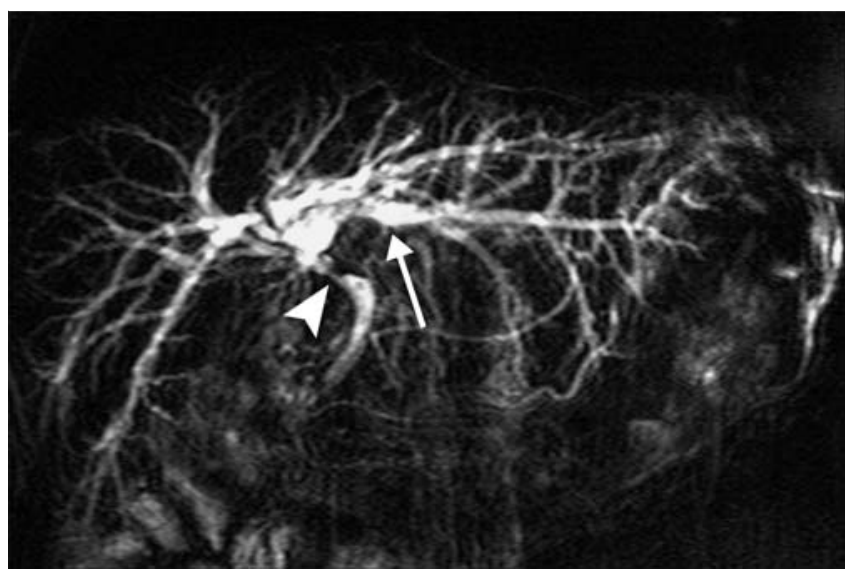

Fig. 2 Three-dimensional reconstruction of preoperative magnetic resonance cholangiopancreatography (MRCP; anteroposterior view) showing the bile duct stricture running close to the hilum (arrow) on the right with consequent dilatation of the left hepatic duct (arrowhead).

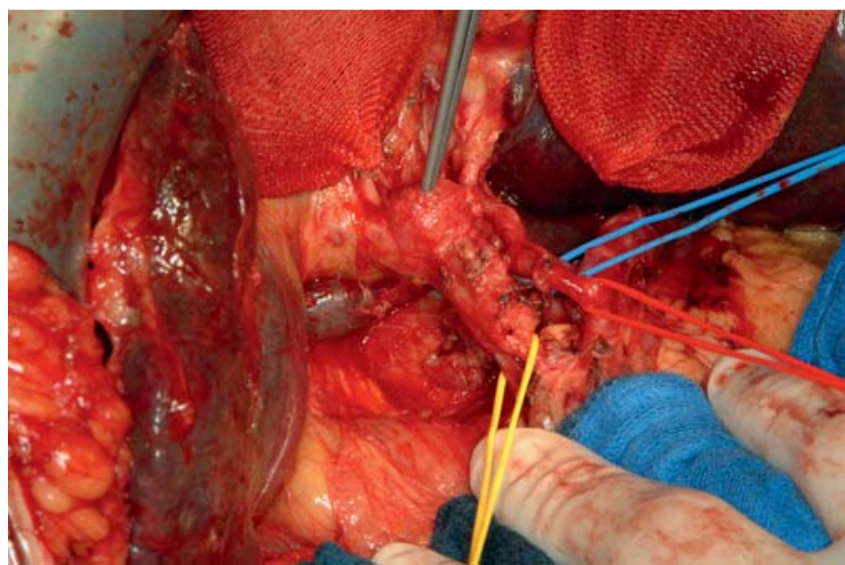

Fig. 3 Intraoperative view after lymphadenectomy at the hepatoduodenal ligament illustrating the tumor (see the grasper). The yellow cord is lifting the common bile duct, the red cord is lifting the hepatic artery and the blue cord is lifting the portal vein.

morbidity. The long-term results of bilioenteric anastomosis for benign strictures are promising [4].
Competing interests: None

Endoscopy_UCTN_Code_CCL_1AZ_2AZ 


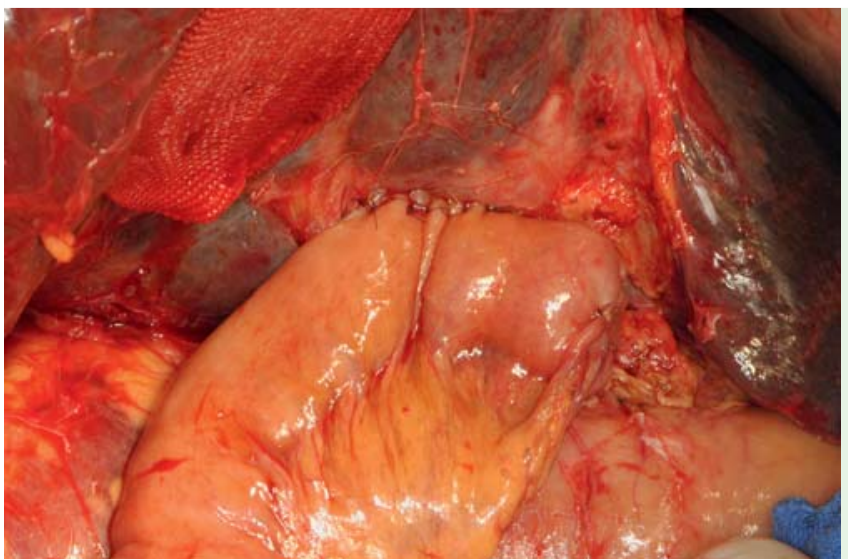

Fig. 4 Intraoperative view following biliodigestive anastomosis.

\section{G. A. Bernhardt, P. Kornprat, W. Schweiger, H. Cerwenka, H. J. Mischinger}

Division of General Surgery, Department of Surgery, Medical University Graz, Graz, Austria troenterol 2009; 15: 4240-4262
3 Nakayama A, Imamura $H$, Shimada $R$ et al. Proximal bile duct stricture disguised as malignant neoplasm. Surgery 1999; 125: 514-521

4 Lillemoe KD. Current management of bile duct injury. Br J Surg 2008; 95: 403-405

\section{Bibliography}

DOI $10.1055 / \mathrm{s}-0029-1244103$

Endoscopy 2010; 42: E148 -E149

(c) Georg Thieme Verlag KG Stuttgart · New York . ISSN 0013-726X

Corresponding author

G. A. Bernhardt, MD

Division of General Surgery

Department of Surgery

Medical University Graz

Auenbruggerplatz 29

8036 Graz

Austria

1 Aljiffry M, Walsh MJ, Molinari M. Advances in diagnosis, treatment and palliation of cholangiocarcinoma: 1990-2009. World J Gas-

2 Gerhards MF, Vos P, van Gulik TM et al. Incidence of benign lesions in patients resected for suspicious hilar obstruction. Br J Surg 2001; 88: 48-51
Fax: +43-316-3854666

gerwin.bernhardt@klinikum-graz.at 